\title{
The Merits of Mannitol in the Repair of Orbital Blowout Fracture
}

\author{
Kyung Jin Shin ${ }^{1}$, Dong Geun Lee ${ }^{1}$, Hyun Min Park ${ }^{1}$, Mi Young Choi ${ }^{2}$, Jin Ho Bae ${ }^{3}$, Eui Tae Lee ${ }^{1}$ \\ Departments of ${ }^{1}$ Plastic and Reconstructive Surgery, ${ }^{2}$ Ophthalmology, and ${ }^{3}$ Anesthesiology, Chungbuk National University College of \\ Medicine, Cheongju, Korea
}

Background One of the main concerns in orbital blowout fracture repair is a narrow operation field, due mainly to the innate complex three dimensions of the orbit; however, a deep location and extensive area of the fracture and soft tissue edema can also cause concern. Swelling of the orbital contents progresses as the operation continues. Mannitol has been used empirically in glaucoma, cerebral hemorrhage, and orbital compartment syndrome for decompression. The authors adopted mannitol for the control of intraorbital edema and pressure in orbital blowout fracture repair.

Methods This prospective study included 108 consecutive patients who were treated for a pure blowout fracture from January 2007 to October 2012. For group I, mannitol was administered during the operation. Under general anesthesia, all patients underwent surgery by open reduction and insertion of an absorbable mesh implant. The authors compared postoperative complications, the reoperation rate, operation time, and surgical field improvement between the two groups.

Results In patients who received intraoperative administration of mannitol, the reoperation rate and operation time were decreased; however, the difference was not statistically significant. The total postoperative complication rates did not differ. Panel assessment for the intraoperative surgical field video recordings showed significantly improved vision in group I. Conclusions For six years, mannitol proved itself an effective, reliable, and safe adjunctive drug in the repair of orbital blowout fractures. With its rapid onset and short duration of action, mannitol could be one of the best methods for obtaining a wider surgical field in blowout fracture defects.

Keywords Orbital fractures / Mannitol / Intraoperative complication
Correspondence: Eui Tae Lee Department of Plastic and Reconstructive Surgery, Chungbuk National University College of Medicine, 410 Sungbong-ro, Heungduk-gu, Cheongju 361-763, Korea

Tel: +82-43-269-6090

FAX: +82-43-269-6387

E-mail: etlee@chungbuk.ac.kr
No potential conflict of interest relevant to this article was reported.

\section{INTRODUCTION}

Fracture of the orbital wall is common, with an incidence ranging from $18 \%$ to $50 \%$ of all craniofacial fractures. Numerous papers have reported on surgical indications, surgical timing, approach options, and reconstruction materials for orbital blowout fracture
[1]. Standards for treatment of orbital blowout fracture are not yet well established. The choice of surgical approach and materials for reduction of orbital blowout fracture depend on the surgeon's experience and preference. Still, surgeons' primary objectives for surgical treatment should be release and replacement of softtissue contents, accurate definition of extent of the fracture, and

Copyright $\odot 2013$ The Korean Society of Plastic and Reconstructive Surgeons

This is an Open Access article distributed under the terms of the Creative Commons Attribution Non-Commercial License (http://creativecommons.org/

licenses/by-nc/3.0/) which permits unrestricted non-commercial use, distribution, and reproduction in any medium, provided the original work is properly cited.

www.e-aps.org 
reconstruction of the orbital wall to its anatomical position in order to restore the original size and conical shape of the orbital cavity while minimizing complications. One of the main obstacles in surgical treatment of a blowout fracture is a narrow field of vision. This narrow surgical field could be related to the complex three-dimensional structure of the orbit, which changes from an rectangular shape anteriorly to a trapezoid shape posteriorly (Fig. 1) [2]. The blowout usually occurs at the two weakest points-the medial wall and/or floor. A bony buttress between those weak points serves as a landmark for categorization of the blowout fracture of both the medial and inferior walls (so-called combined or two-wall fracture) distinguished by whether or not it had collapsed [3]. The orbit contains a number of important structures, including the eyeball, optic nerve, and extraocular muscles and their innervating nerves in this limited complex space. The extent of restriction of the surgical field is also influenced by the severity of the fracture [3], and development of massive edema may also occur during the operation, resulting in the volume expansion of the orbital contents in the limited orbital space, increasingly occluding the field as the operation progresses. The narrow surgical field could end up preventing complete reduction or causing surrounding soft tissue injury, leading to development of serious complications.

Mannitol reduces the volume and pressure of the aqueous humor by extracting fluid from it. It is used for the short-term treatment of acute glaucoma. Similarly, infusions of mannitol are used to lower the elevated intracranial pressure caused by cerebral edema; and it has been used as a medical treatment in retrobulbar hematoma [4]. It produces an osmotic gradient that prevents movement of water from vascular spaces into the cells during membrane pump failure and draws tissue water back into the vascular spaces again. In effect, this reduces the brain and aqueous humor volume and thus lowers intracranial pressure and intraocular pressure. The authors assume that these pharmacologic

Fig. 1. Complex three-dimensional structure of the orbit

A complex three-dimensional orbital structure in computer tomography. The lateral view (A) and coronal view of orbit; from the anterior rectangular shape to the posterior trapezoid shape (B-D).
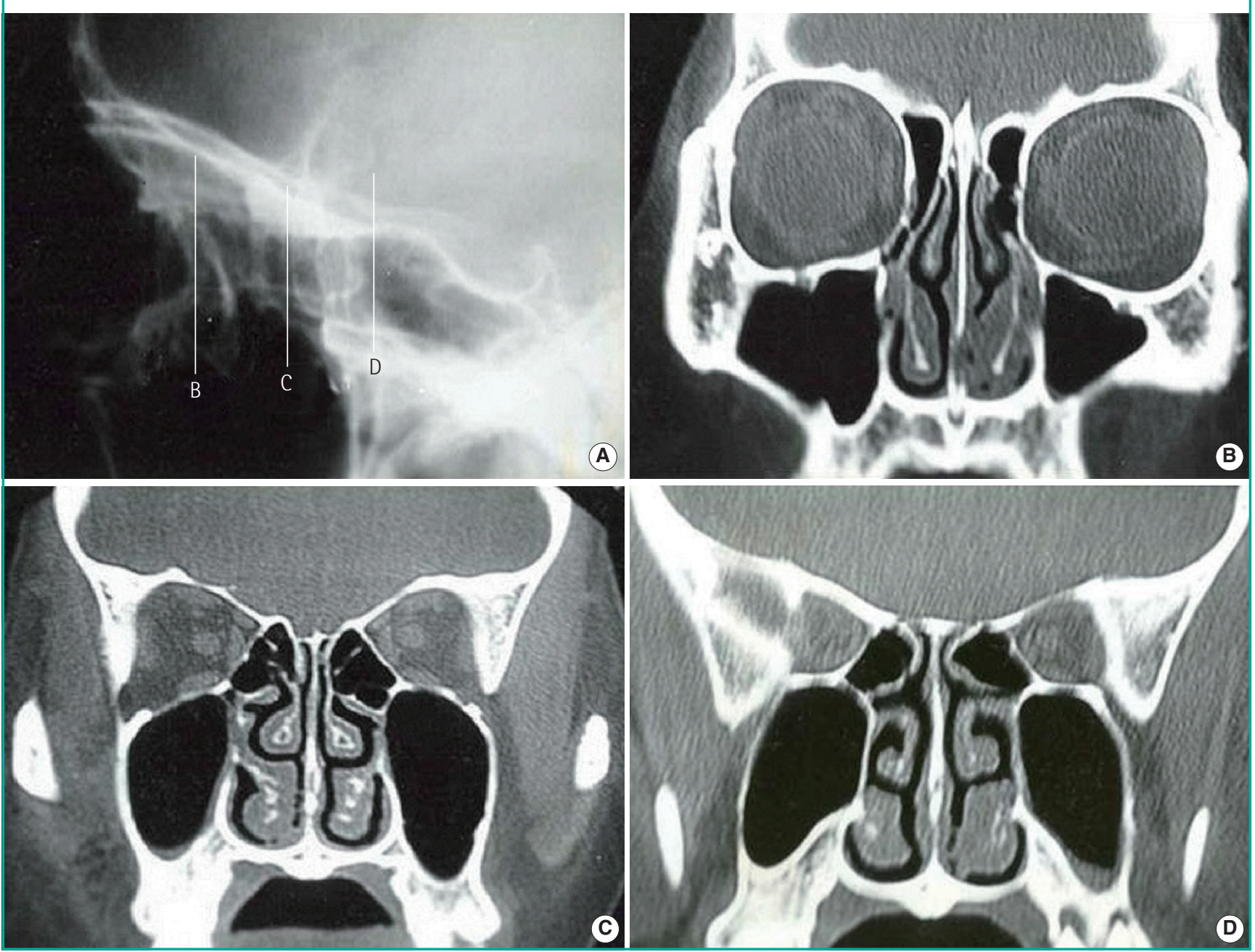
effects of mannitol should also reduce intraorbital pressure and help to improve the surgical field during blowout fracture surgery. We adopted mannitol for the control of intraoperative edema and intraorbital pressure to, in turn, facilitate maintaining a wider operation field and fewer complications associated with poor vision. Therefore, we wanted to prove the usefulness of this pharmacologic agent in blowout fracture surgery.

\section{METHODS}

\section{Subjects}

From January 2007 to October 2012, 108 consecutive patients underwent open reduction and internal fixation for pure orbital blowout fracture. Any cases in which other facial bone fractures occurred were excluded and those in which an absorbable mesh implant was not used as reconstruction material were also excluded. Each subject was randomly allocated to one of the two treatment groups: group I $(n=54)$ received an intravenous infusion of mannitol during the operation and group II $(n=54)$ underwent surgery without mannitol infusion. Randomization was achieved by randomly assigning the subject list to a series

\begin{tabular}{|c|c|c|c|}
\hline Characteristic & Group I $(n=54)$ & Group II $(n=54)$ & $P$-value ${ }^{a)}$ \\
\hline Age (yr) & & & 0.670 \\
\hline Mean \pm standard deviation & $34.20 \pm 13.84$ & $32.68 \pm 13.63$ & \\
\hline Range & $10-70$ & 9-83 & \\
\hline Sex & & & $0.004^{b)}$ \\
\hline Male & $34(63.0)$ & $47(87.0)$ & \\
\hline Female & $20(37.0)$ & $7(13.0)$ & \\
\hline \multicolumn{4}{|l|}{ Fracture type } \\
\hline Medial & $28(51.9)$ & $31(57.4)$ & 0.562 \\
\hline Floor & $12(22.2)$ & $13(24.1)$ & 0.820 \\
\hline Combined & $14(25.9)$ & $10(18.5)$ & 0.355 \\
\hline Diplopia & $8(14.8)$ & $5(9.3)$ & 0.375 \\
\hline Enophthalmos & $20(37.0)$ & $16(29.6)$ & 0.414 \\
\hline Limitating ocular motility & $2(3.7)$ & $2(3.7)$ & 1.000 \\
\hline \multicolumn{4}{|c|}{ 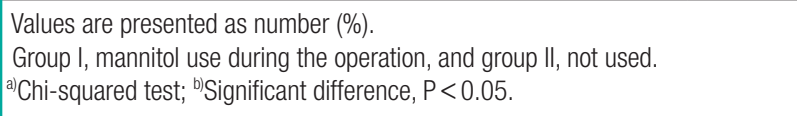 } \\
\hline
\end{tabular}

of computer-generated codes with an equal split between treatment groups. Careful history taking, physical examination, and radiologic and laboratory tests were performed preoperatively. All patients underwent an ophthalmologic evaluation performed by an ophthalmologist preoperatively and one, three, and six months after the operation, if necessary. Enophthalmos was measured directly using the Hertel exophthalmometer. Diplopia was tested in all of the cardinal fields for evaluation of primary and extreme gaze. Age, sex, fracture type (medial/floor/ combined), preoperative and postoperative diplopia, enophthalmos, and ocular motility limitations were recorded (Table 1). An absorbable mesh plate with an average thickness of $2 \mathrm{~mm}$ was used in all of the subjects. Mannitol $(20 \%, 100 \mathrm{~mL})$ was administered to patients in group I through intravenous infusion.

Surgery was performed for the following conditions: limitation of eyeball movement on a forced duction test, moderate or severe displacement of the fractured wall $(>3 \mathrm{~mm}$ ) on computed tomography (CT), and more than $2 \mathrm{~mm}$ of enophthalmos [5]. All of the patients were followed for at least one month after the operation. Postoperative complications, including retrobulbar hematoma, diplopia, remnant enophthalmos, and scarring, as well as the reoperation rate were compared between the two groups. The operation duration and operation field of the two groups were also compared. For evaluation of the operation field with or without intraoperative administration of mannitol, we used a visual analogue scale. Standardized digital video recordings before and after infusion of mannitol (group I, 25 pairs) and Hartman's solution (group II, 25 pairs) were loaded into the computer. Panels were also given an explanation of how to use a visual analogue scale to score an operation field. That is the basis of the evaluation that showed pictures with a panel (Fig. 2). Video recordings were displayed in a random singleblind fashion. When the individual images were displayed, the assessors would document and be asked to confirm a visual analogue scale score. The operation field was rated on a visual analogue scale between 1 (totally obstructive view) and 10 (totally open view). Enhancement of the operation field after infusion

\section{Fig. 2. Visual analogue scale of the operation field of the orbit}

Operation field of the orbit from (A) 0 points to (F) 10 points (0-2-4-6-8-10). Figure (F) shows the best operation field.
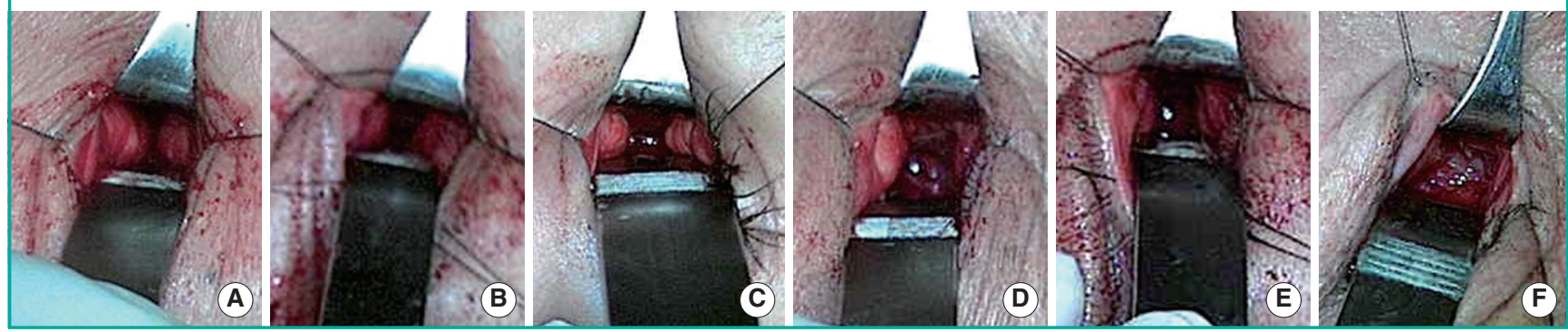
of mannitol or Hartman's solution was compared between the two groups.

\section{Operation method}

Under general anesthesia, a patient was placed in supine position, followed by insertion of a urethral catheter and skin preparation. Hourly urine monitoring and electrolyte tests were performed during the operation. A medial transconjunctival incision [6-9], inferior transconjunctival incision, and combination of them were used for fracture of the medial wall, floor, and both walls, respectively. The size and shape of the orbital defect were measured and an absorbable polylactic acid-polyglycolic acid sheet implant was trimmed according to the bony defect and inserted. During the subperiosteal dissection and insertion of reparative material, $20 \%$ mannitol $(100 \mathrm{~mL})$ was infused intravenously at a rate of $5 \mathrm{~mL} / \mathrm{min}$ (total of 20 minutes). The orbital soft tissue shrank, ensuring a much wider operative field. In 50 patients, standardized digital video recordings were taken. The recordings were made just before administration of mannitol and in the middle of mannitol infusion (10 minutes after the start of infusion) in 25 patients and administration of Hartman's solution in another 25 patients. The video recordings were performed in standardized fashion at $50 \mathrm{~cm}$ distance using a builtin camera in a head lamp (SurgiCam Digital camera system, General Scientific Corp., Ann Arbor, MI, USA).

The authors performed the forced duction test to confirm the mobility of the eyeball and checked the pupil size, and direct and indirect light reflex, in order to avoid inadvertent compression of the optic nerve before closure of periosteum and mucosal incisions.

On postoperative day 1 , the patients were examined for extraocular movement limitations, symptomatic retrobulbar hemorrhage, severe remaining orbital herniation and enophthalmos on CT, and emergency reoperation was performed. Otherwise, reoperation was considered for remaining enophthalmos an three weeks after initial operation.

\section{Statistical analysis}

Bivariate comparison of the demographics, complication rates, reoperation rates, and operation time comparison turtle the mannitol use and mannitol non use groups were performed using a chi-squared test for categorical variables. We used a paired t-test to compare a sample group's scores before and after mannitol or Hartman's solution administration. We test whether there is a significant difference in the number of edits the subjects' complete in group 1 (mannitol group) and group 2 (Hartman's solution group). Comparison of enhancement of the operation field was processed. Statistical significance was recognized when the P-value was 0.05 or less. All data manipulation and statistical calculations were performed using SPSS ver. 20.0 (IBM Corp., Armonk, NY, USA).

\section{RESULTS}

Among 108 patients who underwent repair of a blowout fracture, the same number, 54 patients, were allocated to each group: group I (mannitol use group) and group II (mannitol non-use group) in randomized fashion. A mean age of 34.20 years, a proportion of males to females of 34 to 20, and fracture type distribution ratio of medial wall fractures to floor fractures to combined fractures as 28:12:14 were noted in group I. Preoperatively, group I included 30 symptomatic patients (20 enophthalmic patients, eight diplopia patients, and two patients with limitation of ocular movement). The mean age of 32.68 years, proportion of males to females 47 vs. 7 , and fracture type distribution ratio of medial wall fractures to floor fractures to combined fractures as 31:13:10 were noted in group II. In group II, a total of 23 symptomatic patients (16 enophthalmic patients, five diplopia patients, and two patients with limitation of ocular movement) were recorded preoperatively. Therefore, no significant differences in these parameters were observed between the two groups, except for the sex distribution ratio (Table 1). In the mannitol use group, there were seven cases (13.0\%) of postoperative complications: one case of diplopia (1.9\%) and six remnant enophthalmos (11.1\%). Reoperation was performed in one patient $(1.9 \%)$ in whom enophthalmos and diplopia occurred concurrently. That patient had diplopia in upward gaze and enophthalmos of $2 \mathrm{~mm}$ at 18 days after the initial operation. The previous implant was removed and replaced with a new implant after further reduction. The symptoms showed improvement; however, diplopia remained at the left upper extreme gaze at three months after reoperation. In the mannitol non-use group, there were seven cases (13.0\%) of postoperative complications: one of retrobulbar hematoma (1.9\%), three of diplopia (5.6\%), two remnant enophthalmos (3.7\%), and one case of operative scar problem (1.9\%). Among them, five patients (9.3\%) underwent reoperation. One patient had lateral dystopia and limitation of ocular motility at postoperative day 1 and diffuse retrobulbar hemorrhage without muscle entrapment was observed on CT. After reoperation for the decompression of intraorbital pressure, extraocular muscle movement showed improvement; however, diplopia remained at the lateral and inferior gaze, and impaired visual acuity continued at 1 year postoperatively. The overall incidence of preoperative and postoperative complications was not significantly different; however, preoperative symptomatic patients (30 vs. 23) 
had a tendency to be more frequent in the mannitol group and some serious immediate postoperative complications requiring reoperation ( 1 vs. 5) were more frequent in the non-mannitol group $(\mathrm{P}=0.093)$. The operative time was shorter in the mannitol use group ( $88.5 \pm 32.1$ minutes) compared to the mannitol non-use group (90.7 \pm 38.9 minutes) (Table 2). The fracture type distribution ratio of medial wall to floor of combined fracture as 11:11:3, as noted in the video recording of the Mannitol group, and 8:2:15 were noted in a video recording of Hartman's solution group. No significant differences in each fracture type

\begin{tabular}{|c|c|c|c|}
\hline Characteristic & Group I $(n=54)$ & Group II $(n=54)$ & $P$-value ${ }^{a)}$ \\
\hline Complications (\%) & $7(13.0)$ & $7(13.0)$ & 1.000 \\
\hline Retrobulbar hematoma & $0(0)$ & $1(1.9)$ & 0.315 \\
\hline Diplopia & $1(1.9)$ & $3(5.6)$ & 0.308 \\
\hline Remnant enophthalmos & $6(11.1)$ & $2(3.7)$ & 0.142 \\
\hline Minor complication & $0(0)$ & $1(1.9)$ & 0.315 \\
\hline Reoperation & $1(1.9)$ & $5(9.3)$ & 0.093 \\
\hline \multicolumn{4}{|l|}{ Operation time (min) } \\
\hline Mean \pm standard deviation & $88.52 \pm 32.05$ & $90.74 \pm 38.90$ & \\
\hline Range & 30-180 & $35-170$ & 0.324 \\
\hline
\end{tabular}

Group I, mannitol use during the operation, and group II, not used.

a) Chi-squared test; : Significant difference, $P<0.05$. were observed between before and after the visual analogue scale (VAS) score, because the number of each case was too small and the numbers of each case through classification were not the same. Therefore, we did not distinguish among these details, but evaluated the cases before and after scoring together. The visual analogue scale score before and after mannitol administration was shown to be markedly different, and this had statistical significance. However, no significant differences in Hartman's solution group were observed between before and after the VAS score (Table 3). There was no occurrence of immediate or delayed recipient complications of infection, extrusion, displacement, or foreign body reaction related to the implant. There was no occurrence of major drug related complications in

Table 3. Visual analogue scale of the operation field of the orbit

\begin{tabular}{|c|c|c|c|c|c|}
\hline \multirow{2}{*}{ Variable } & \multicolumn{3}{|c|}{ Score } & \multirow{2}{*}{$t$} & \multirow{2}{*}{ P-value } \\
\hline & Before & After & Difference & & \\
\hline Group I (n=25) & $3.36 \pm 2.63$ & $6.96 \pm 3.35$ & $3.60 \pm 2.08$ & 8.647 & $0.000^{b)}$ \\
\hline Group II $(n=25)$ & $5.36 \pm 2.81$ & $5.60 \pm 2.65$ & $0.24 \pm 0.66$ & 1.809 & 0.083 \\
\hline
\end{tabular}

\section{Fig. 3. A case of intraoperative mannitol use}

A 46-year-old-female with a blowout fracture in the right medial and inferior wall. (A) Preoperative photo with a worm's eye view shows moderate enophthalmos in the involved eye. (B) Two months after the operation, the enophthalmos had been resolved.
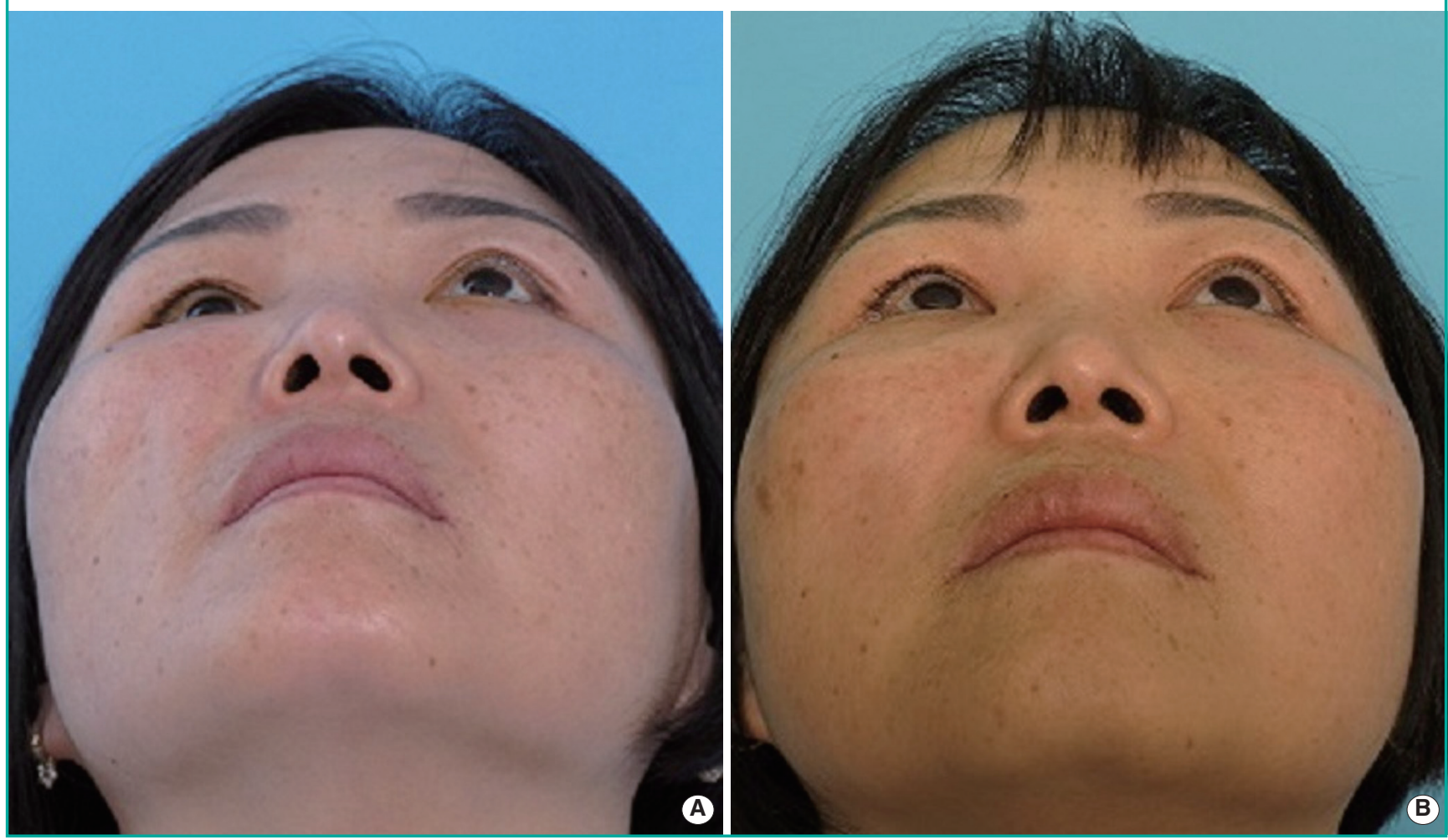
the central nervous, cardiovascular, pulmonary, or renal systems. Other minor drug related side effects such as headache, nausea, vomiting, and abdominal cramps were also not present. Some representative cases are shown in Fig. 3.

\section{DISCUSSION}

One of the main concerns in surgical treatment of a blowout fracture is the narrow field of vision. Massive edema may occur during the operation and the resultant volume expansion of the orbital contents in the compact orbital space occludes the field increasingly as the operation goes on. Pure orbital blowout fractures are classified as medial wall fractures, inferior wall fractures, and combined medial and inferior wall fracture according to the involved area. The greater the fracture area is, the more extensive the soft tissue swelling and resultant surgical field obstruction would be. The authors applied 20\% mannitol in order to overcome these difficulties. In the author's opinion, use of this method resulted in a wider surgical field and thus facilitated repair. In particular, non-experienced surgeons who are not familiar with repair of blowout fracture could benefit most from use of mannitol. Mannitol can cause central nervous system derangement, pulmonary edema, and dehydration [10]. Acute expansion of the extracellular fluid volume engendered by osmotic diuretics increases the work load of the heart. Patients in cardiac failure are particularly susceptible, and development of pulmonary edema may occur. Therefore, they should not be treated with these drugs. Mild hyperkalemia is often observed; however, an intolerable potassium elevation is not likely, except in patients with diabetes, adrenal insufficiency, or severely impaired renal function. Careful history taking, physical examination, and radiologic and laboratory tests should be performed in order to rule out these conditions. In this study, patients were usually healthy (American Society of Anesthesiologists classification 1 or 2) and no patient was excluded in this regard. Severe volume depletion and hypernatremia may result from prolonged administration of mannitol unless lost sodium and water are replaced. However, we were able to obtain the target effect with the use of a much smaller dose $(20 \mathrm{~g})$ than in the case of intracranial decompression ( 1 to $3 \mathrm{~g} / \mathrm{kg}$ ) for a short duration (20 minutes) [11]. An intraoperative electrolyte test and hourly urine monitoring via a Foley catheter were performed in order to guarantee safety. Quantitative analysis of the surgical field enhancement after administration of mannitol is difficult to measure in practice. Direct orbitonometry is an invasive examination that could cause iatrogenic retrobulbar hematoma or intraorbital organ injury on an otherwise healthy organ [12]. Therefore, due to ethical and legal problems, we rejected the idea of direct measurement of orbital pressure in human. Animal experiments were attempted; however, animals with an anatomical structure similar to the orbit of humans are rare, except apes. Normal human orbital pressure is 4 to $6 \mathrm{~mm} \mathrm{Hg}$, a very low value [13]. Animals' orbital pressure is even smaller than that of humans, and the pressure monitor for animals cannot be calibrated. Therefore, the effect of mannitol administration was evaluated through panel surveys on the clinical video recordings of a pre-mannitol and post-mannitol view during the operation. However, this method is limited by the subjective nature of the evaluation. If we could devise an objective method for measuring the amount of surgical field widening in the future, the reliability of mannitol could gain more support. For six years, mannitol has proven itself an effective, reliable adjunctive drug without side effects in the repair of orbital blowout fractures. With its rapid onset and short duration of action, mannitol could be one of the best methods for obtaining a wider surgical field in a complex three-dimensional fracture defect, especially in cases of extensive blowout fracture for surgeons with limited experience.

\section{REFFERENCES}

1. Kwon J. Update in treatment of orbital blowout fractures. Korean J Otorhinolaryngol-Head Neck Surg 2011;54:31723.

2. Manson PN. Facial fractures. In: Mathes SJ, Hentz VR, editors. Plastic surgery. 2nd ed. Philadelphia: Saunders; 2006. p.264-305.

3. Burm JS, Chung CH, Oh SJ. Pure orbital blowout fracture: new concepts and importance of medial orbital blowout fracture. Plast Reconstr Surg 1999;103:1839-49.

4. Brody TM, Larner J, Minneman KP. Brody's human pharmacology: molecular to clinical. St. Louis: Mosby; 1998.

5. Rodriquez ED, Losee JE, Neligan PC. Plastic surgery Philadelphia: Saunders; 2012.

6. Chen CT, Chen YR, Tung TC, et al. Endoscopically assisted reconstruction of orbital medial wall fractures. Plast Reconstr Surg 1999; 103:714-20.

7. Shorr N, Baylis HI, Goldberg RA, et al. Transcaruncular approach to the medial orbit and orbital apex. Ophthalmology 2000;107:1459-63.

8. Graham SM, Thomas RD, Carter KD, et al. The transcaruncular approach to the medial orbital wall. Laryngoscope 2002;112:986-9.

9. Mun GH, Song YH, Bang SI. Endoscopically assisted transconjunctival approach in orbital medial wall fractures. Ann Plast Surg 2002;49:337-43.

10. Goodman LS, Gilman AG, Brunton LL, et al. Goodman \& 
Gilman's the pharmacological basis of therapeutics. New York: McGraw-Hill Medical; 2011.

11. Yamada KA, Awadalla S. Neurologic disorders. In: Green GB, Harris IS, Lin GA, et al., editors. The Washington manual of medical therapeutics. 31st ed. Philadelphia: Lippincott Williams \& Wilkins; 2004. p.532-537.
12. Matsen FA 3rd, Winquist RA, Krugmire RB Jr. Diagnosis and management of compartmental syndromes. J Bone Joint Surg Am 1980;62:286-91.

13. Zhou H, Fan X, Xiao C. Direct orbital manometry in normal and fractured orbits of Chinese patients. J Oral Maxillofac Surg 2007;65:2282-7. 